\title{
Sight for blind mice
}

Collaborators at two major research institutions in the US have shown that gene therapy can target cone cells and rescue electrical response and visual acuity in a mouse model of achromatopsia, a disorder causing hereditary blindness in roughly 1 of every 30,000 humans.

Achromatopsia is the result of cone vision loss and leads to nearly complete color blindness and poor central vision in most of those who suffer from the disease.

Mice carrying a mutation in the gene Gnat2, which encodes a guanine nucleotide binding protein, have a phenotype similar to that of humans with complete achromatopsia. William W. Hauswirth of the University of Florida College of Medicine (Gainesville) and his colleagues at The Jackson Laboratory (Bar Harbor, $\mathrm{ME}$ ) used an adenovirus vector to deliver wildtype Gnat 2 under the control of a conespecific promoter into the subretinal space of mutant mouse eyes roughly one month after birth. At 1-2 months after treatment, 19 of 21 eyes responded to the gene therapy, and 17 of those showed electroretinogram (ERG) signals comparable to wildtype mice (Nat. Med., doi:10.1038/ nm1596, published online 21 May). In addition, 18 of 21 treated eyes continued to have measurable ERG signals 5-6 months after treatment, 17 of which were still in the normal range. The same gene therapy methodology also rescued the ERG signals in 9-month-old mice.

The researchers used a
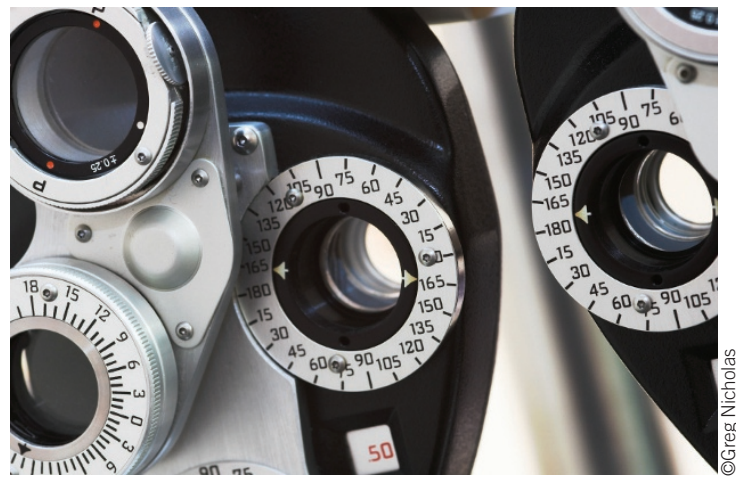

behavioral test to determine

whether the ERG restoration was associated with changes in visual acuity. They found that visual acuity improved in eyes treated with wildtype Gnat 2 but not in untreated eyes. In fact, mean visual acuity was not significantly different in treated versus wildtype eyes.

To date, this is the first time cone-targeted gene therapy has been used to restore visual function in an animal model of achromatopsia. As such, this research has important implications for treating various forms of human blindness. "Clinically, this work may directly impact human achromatopsia in a few years," Hauswirth tells Lab Animal. "More broadly, this work opens up the possibility of helping cones survive the most prevalent causes of blindness in the US-age-related macular degeneration, diabetic retinopathy, and retinitis pigmentosa-none of which initially affect cones but all of which eventually lead to cone loss, the final event leading to blindness." Hauswirth and colleagues are now working to confirm this approach in a dog model that represents the most common form of human achromatopsia.

Monica Harrington

\section{A NEW MODEL FOR MUSCLE CANCER}

A team of researchers have developed a new animal model for rhabdomyoscarcoma (RMS), a deadly form of childhood muscle cancer. Scientists may have already succeeded in using the new zebrafish model to identify the group of cells that makes this form of cancer so resilient and dangerous.

RMS is diagnosed in more than 250 new patients each year in the US. Because of the aggressive nature of this kind of cancer, patients suffering from RMS must undergo an arduous treatment regime that often fails. Of those with metastatic disease at the time of diagnosis, less than $25 \%$ survive longer than five years.

Researchers have developed several murine models of RMS that have aided understanding of this disease, but these models require complicated mouse breeding and do not develop the necessary tumors quickly. To circumvent these problems, David M. Langenau and colleagues at the Children's Hospital Boston (MA) created a zebrafish model of embryonal RMS, the most common RMS subtype. By 80 days of age, half of the zebrafish develop embryonal RMS.

Langenau and his coworkers used microarray analysis and a technique called gene set enrichment analysis on the new model to find similarities in the gene expression profile of the zebrafish and human embryonal RMS cancer cells (Genes Dev., doi:10.1101/ gad.1545007, published online 17 May). Their data strongly suggest that the molecular machinery responsible for embryonal RMS is conserved between the two species-evidence that the zebrafish will serve as a reliable model for the human disease.

In the same study, Langenau also took tumor cells from embryonal RMS zebrafish, sorted them by cell type, and transplanted them serially into healthy zebrafish. They found that a subset of tumors cells can recapitulate the cancer in healthy zebrafish, as though these cells are a kind of embryonal RMS 'stem cell'. The researchers noted that these tumor stem cells are similar in molecular composition to normal muscle satellite cells, a finding that suggests embryonal RMS may originate in muscle satellite cells.

"The ultimate goal [of the zebrafish model] would be to identify pathways that have gone understudied in RMS, ultimately leading to new ideas of how better to treat patients with this debilitating disease," Langenau tells Lab Animal. He continues, "We are hopeful that further interrogation of the pathways we identified in our study will lead to new lines of treatment."

Owen Young 